\title{
Distribution of mucosal pathology and an assessment of colonic phenotypic change in the pelvic ileal
} reservoir

\author{
N A Shepherd, C J Healey, B F Warren, P I Richman, W H F Thomson, S P Wilkinson
}

\begin{abstract}
The mucosa of the pelvic ileal reservoir undergoes adaptive changes - inflammatory, architectural, and metaplastic - on exposure to the faecal stream. Twenty three quadruple loop ileal pouches constructed for ulcerative colitis (20 patients) and familial adenomatous polyposis (FAP) (three patients) were studied. No patient fulfilled clinical, endoscopic, or histopathological criteria for pouchitis. Standard duplicate biopsy specimens were taken from the proximal limb, the anterior wall, the posterior wall, and the body of the reservoir. An established scoring system was used and showed a highly significant increase in inflammatory scores in posterior wall biopsy specimens compared with those from the anterior wall. These results suggest that the adaptive changes are the direct result of contact with static faecal contents. One patient only showed significant inflammation in the proximal limb. There was no evidence of mucosal prolapse in any anterior wall biopsy specimen. Patients with colitis showed substantially more inflammatory and architectural changes than those with FAP. Ninety six per cent of pouches showed some colonic phenotypic expression as defined by mucin histochemical and PR 3A5 immunohistochemical studies. Our results suggest, however, that there may not be complete colonic metaplasia and that the mucin changes and other phenotypic alterations may represent a non-specific response to pouch inflammation and not a prerequisite for the development of pouchitis. The focal nature of the inflammatory and architectural changes, which may be the result of direct contact with static faecal residue, are clearly shown. A single random biopsy specimen of pouch mucosa is of limited value in assessing pathological changes and screening for potential neoplastic change within the reservoir.
\end{abstract}

(Gut 1993; 34: 101-105)

Restorative proctocolectomy with ileal reservoir has become established as an important surgical alternative for patients with severe ulcerative colitis, familial adenomatous polyposis (FAP), and other pancolic conditions such as idiopathic megacolon. The formation of a pelvic ileal reservoir results in pathological changes in the ileal mucosa in most patients regardless of the initial diagnosis.' These include alterations of a chronic nature such as chronic inflammation, villous atrophy, and crypt hyperplasia, while more acute changes such as a neutrophil infiltrate and ulceration are less common and, when severe, equate with the clinical condition of pouchitis. ${ }^{12}$ Pelvic ileal reservoirs have been shown to express colonic phenotypic changes and it has been suggested that the reservoir mucosa undergoes complete colonic metaplasia. This concept has been supported by histopathological, ${ }^{13}$ histochemical, ${ }^{1+5}$ immunohistochemical, ${ }^{467}$ and ultrastructural ${ }^{8}$ studies. While focality of pathological changes has been observed, ${ }^{69}$ very little is known about the distribution of inflammatory and metaplastic changes in the reservoir and the proximal limb. These distributions were studied in an unselected patient group with pelvic ileal reservoirs constructed for both ulcerative colitis and FAP.

\section{Methods}

Twenty three patients who had undergone restorative proctocolectomy with ileal reservoir were included in the study. In all patients a quadruple loop (W) pouch had been constructed by the same surgeon (WHFT) according to standard techniques. ${ }^{10}$ On hospital attendance, each patient completed a questionnaire and a consent form. The questionnaire comprised symptomatic assessment, including frequency of bowel action; current medication; and degree of anal continence. Endoscopy was performed with an Olympus OSF $60 \mathrm{~cm}$ flexible sigmoidoscope under sedation with intravenous benzodiazepine as necessary. Preparation of the reservoir for endoscopy was performed with a phosphate enema 30 minutes before the examination.

Endoscopic examination of the ileal mucosa included a macroscopic assessment of the pouch mucosa and entry into the afferent ileal limb. Two biopsy specimens were taken using standard biopsy forceps from four consistent sites:

(a) The afferent limb, at least $5 \mathrm{~cm}$ proximal to the pouch itself;

(b) The anterior wall $5 \mathrm{~cm}$ above the pouchanal anastomosis;

(c) The posterior wall $5 \mathrm{~cm}$ above the pouchanal anastomosis;

(d) The body of the pouch. These biopsies were taken from the centre of the pouch, on the posterior wall, $10 \mathrm{~cm}$ above the pouch-anal anastamosis.

Biopsy tissues were fixed in buffered formalin and processed routinely through paraffin wax, ensuring optimal orientation at the embedding stage. Sections $(5 \mu)$ were cut at six levels and stained with haematoxylin and eosin, periodic acid Schiff, and high iron diamine alcian blue (HIDAB), the latter with strict $\mathrm{pH}$ con- 
TABLE I Clinical data for the 23 patients

\begin{tabular}{|c|c|}
\hline Age (mean (range)) & $39 \cdot 5(20-63)$ \\
\hline $\operatorname{Sex}(M: F)$ & $12: 11$ \\
\hline Original diagnosis (UC:FAP) & $20: 3$ \\
\hline $\begin{array}{l}\text { Operation type (one stage:two } \\
\text { stage) }\end{array}$ & $5: 18$ \\
\hline $\begin{array}{l}\text { Duration of functioning pouch } \\
\text { (mean (range)) }\end{array}$ & $39 \cdot 5$ Months (6-75 months) \\
\hline Medication & $\begin{array}{l}\text { Codeine phosphate (3); imodium } \\
\text { (3); both (1); no medication } \\
\text { (16) }\end{array}$ \\
\hline $\begin{array}{l}\text { Frequency of defecation (mean } \\
\text { (range) per } 24 \text { hours) }\end{array}$ & $6 \cdot 2(2 \cdot 5-12 \cdot 5)$ \\
\hline Nocturnal defecation & Never (10); regularly (13) \\
\hline Anal incontinence & $\begin{array}{l}\text { Never }(14) \text {; occasionally }(8) \\
\text { regularly }(1)\end{array}$ \\
\hline
\end{tabular}

$\mathrm{UC}=$ ulcerative colitis $\mathrm{FAP}=$ familial adenomatous polyposis.

trol. ${ }^{11}$ Immunohistochemistry was performed using the streptavidin-biotin complex (ABC) method with the primary monoclonal antibody PR 3A5. ${ }^{12}$ PR $3 A 5$ is known to bind to an epitope on the O-acetylated sialomucin molecule and is specific, in normal mucosa, to colorectal type epithelium. ${ }^{12}$ For positive controls, sections containing rectal carcinoma and normal mucosa were employed and for negative controls the primary antibodies were excluded.

Standard haematoxylin and eosin stained preparations were scored according to the inflammatory scale previously established for use in reservoir mucosal biopsy tissue. ${ }^{12}$ In this scale both acute (neutrophil polymorph infiltrate $0-3$ : ulceration 0-3) and chronic (chronic inflammation 0-3: villous atrophy 0-3) changes are scored. Correlation was sought between the mean and distribution of inflammatory scores and the clinical data shown in Table I. Evidence of other pathological changes, such as mucosal prolapse, mucosal ischaemia, pseudopyloric metaplasia, or granulomas ${ }^{69}$ was also noted. HIDAB stained slides and PR 3A5 immunostains were assessed for sulphomucin (staining brown-black) and PR 3A5 immunoreactivity on the scale shown in Table II.

Approval for the study was granted by the Gloucester Health Authority Ethical Committee.

\section{Results}

Table I shows clinical data for all 23 patients. Figure 1: The distribution of total pathological scores for the four biopsy sites.

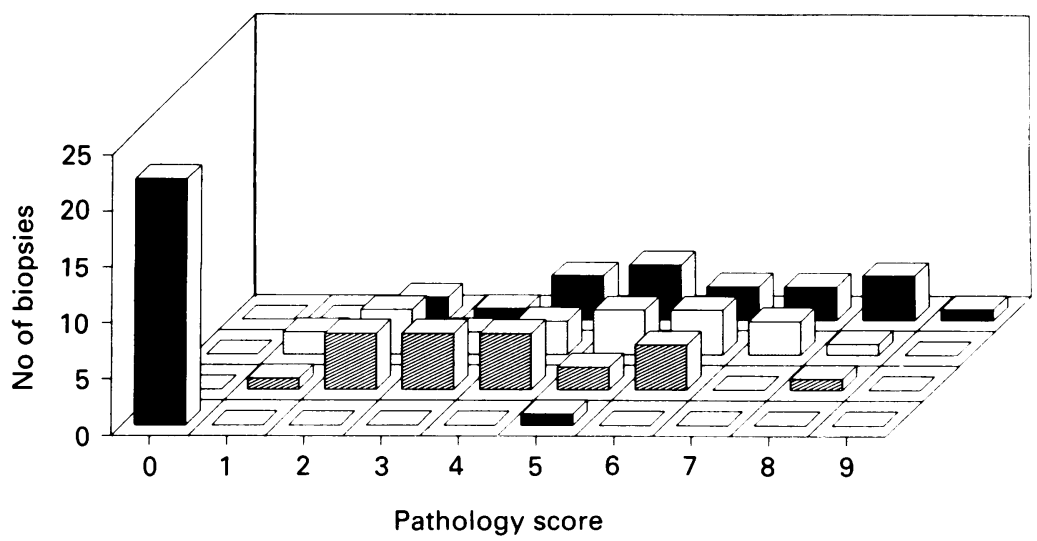

ulcerative colitis in 20 patients and FAP in three. The diagnosis of ulcerative colitis was confirmed by review of all 20 proctocolectomy specimens. In 18 patients the operation had been a two stage procedure with a covering ileostomy being closed some three months after primary surgery while in five patients a one stage restorative proctocolectomy with ileal reservoir was performed with the ileal reservoir becoming functional at the time of proctocolectomy. In all patients the pouch had been fully functional for at least six months. None of the patients fulfilled the clinical, endoscopic, and histopathological criteria for pouchitis. ${ }^{1314}$

The distribution of pathological scores for the four biopsy sites is shown in Figure 1. Scores were highest for posterior wall biopsy specimens (mean (SD), 5.5 (1.9)), intermediate for body specimens $(4 \cdot 2(1 \cdot 7))$, and lowest for anterior wall specimens $(3.9(1.6))$. There was a highly significant difference between anterior and posterior wall pathological scores (Wilcoxon $\mathrm{p}=$ 0.001 ) (Figs $2 \& 3$ ). There was no correlation between these inflammatory scores or their distribution and age, sex, duration of the functioning reservoir, symptomatology, or treatment. Inflammatory scores in biopsy specimens from all sites were consistently lower in the three FAP patients than in colitis patients (Fig 3 ). Thus, the level of inflammatory scores correlated only with the original diagnosis and no other clinical parameter affected the intensity of inflammation or its distribution.

Only one patient showed significant inflammation in the proximal limb. This was a 29 year old man with a 10 year history of ulcerative colitis who had undergone restorative proctocolectomy five years previously. There was no evidence of backwash ileitis in the proctocolectomy specimen. Analysis of the anterior wall biopsy specimens showed none of the characteristic histopathological features of mucosal prolapse. No granulomas were evident in any tissue, there were no pathological features of mucosal ischaemia, and no pseudopyloric metaplasia was observed. ${ }^{9}$

The results of the histochemical and immunohistochemical assessments are shown in Table II and the comparative expression of these colonic type markers is shown in Table III. Although there was clear evidence of a variation in inflammatory and architectural changes in the body,

TABLE II Results of histochemical and immunohistochemical analysis

\begin{tabular}{llc}
\hline & Sulphomucin & PR 3A5 \\
\hline No staining & 9 & 5 \\
Focal $(<5 \%$ of goblet cells $)$ & 2 & 2 \\
Focal strong $(5-25 \%)$ & 5 & 11 \\
Extensive staining $(>25 \%)$ & 7 & 5 \\
\hline
\end{tabular}

TABLE III Comparison of sulphomucin and PR $3 A 5$ expression

\begin{tabular}{llll}
\hline & \multicolumn{2}{l}{ Sulphomucin } & \\
\cline { 2 - 4 } & $-v e$ & $+v e$ & Total \\
\hline PR 3A5: & & 4 & \\
-ve & 1 & 10 & 5 \\
+ve & 8 & 14 & 18 \\
Total & 9 & & 23 \\
\hline
\end{tabular}


A

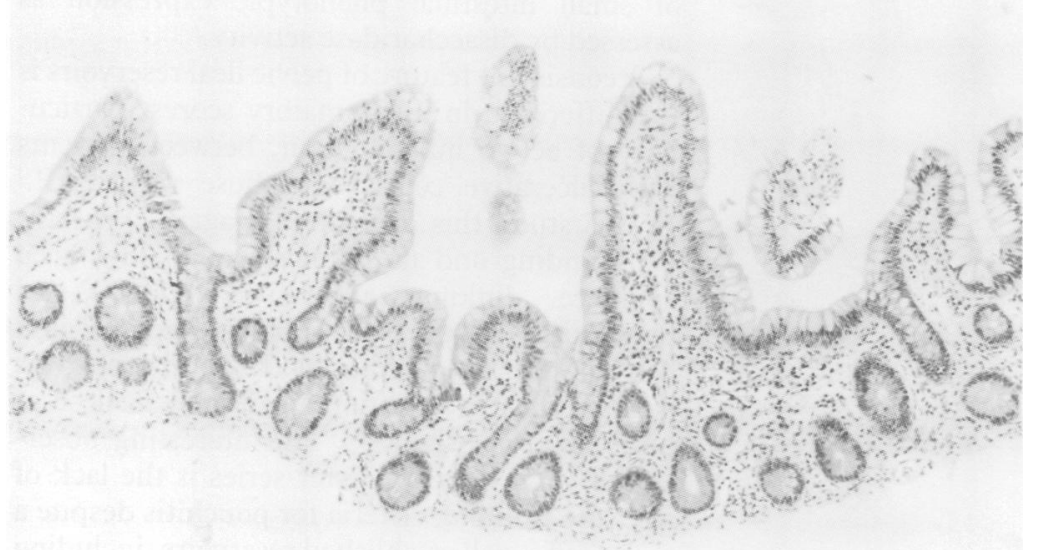

B

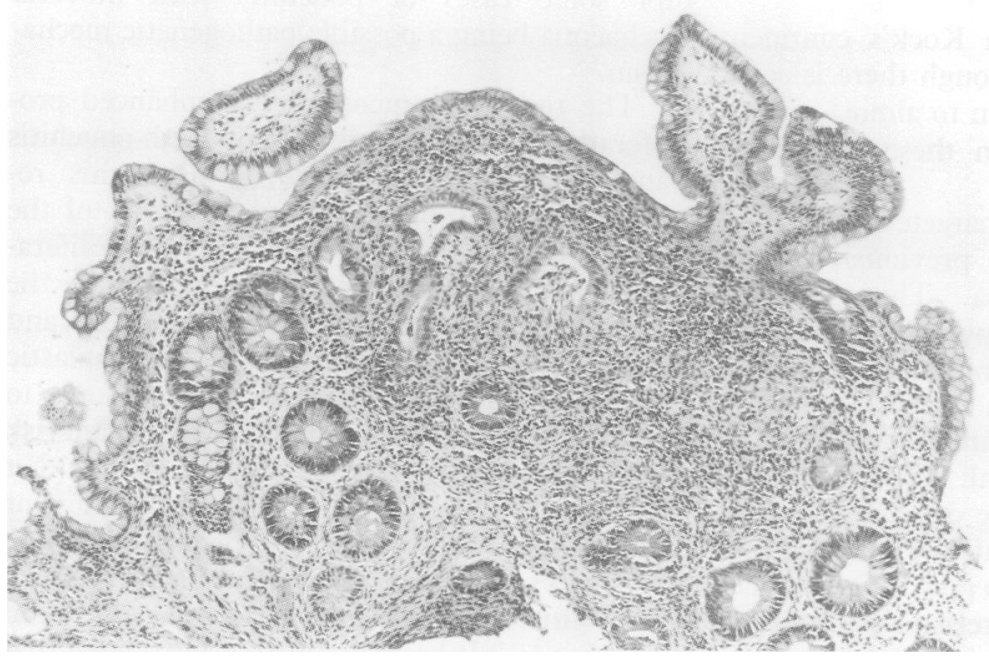

Figure 2: Anterior wall biopsy $(A)$ and posterior wall biopsy $(B)$ from a 28 year old man with previous ulcerative colitis. The anterior wall biopsy shows only minor architectural and chronic inflammatory changes (score 2) while the posterior wall biopsy shows partial villous atrophy, diffuse chronic inflammation, and activity in the form of disruptive crypt abscesses (score 6).

(Haematoxylin and eosin original magnification $\times 100$.)

mens, no consistent differences could be shown for these histochemical and immunohistochemical markers between the three pouch biopsy sites. In general, the same level of expression was present at all sites within the reservoir, although the only afferent limb biopsy specimen to show such expression was the single case with significant inflammatory and architectural abnormality. Although $61 \%$ of reservoirs showed sulphomucin expression and 78\% demonstrated PR 3A5 immunoreactivity (Fig 4), those from the anterior wall cannot be explained by any clinical factor. It is unlikely that technical factors are responsible for the differences. Although more tension may be applied to the lower posterior wall of the reservoir than to the anterior wall during construction, one would expect to show greater differences in scores for these sites in relatively recently constructed reservoirs compared with those that had been in situ for several years, and the pathological changes would also be expected to be more like those of mucosal ischaemia. In the functioning reservoir the liquid contents of the reservoir are in contact with the posterior wall mucosa in both the prone and standing positions while the anterior wall mucosa is somewhat protected by the presence of gas within the lumen. Our thesis that the mucosal adaptive changes are related to static faecal residue contact is very much supported by the intermediate pathological scores for the body mucosal biopsy specimens and the almost universal lack of noticeable change in the proximal limb specimens.

Various microbiological changes have been observed in pelvic ileal reservoirs, although these have not been consistent. ${ }^{31516}$ Nevertheless, inverse correlation has been shown between volatile fatty acids reflecting anaerobic bacterial acitivity and altered mucosal architecture. ${ }^{16}$ These findings suggest that anaerobic bacterial activity has a protective effect on the ileal mucosa. It seems likely that it is the ratio of anaerobes to pathogenic aerobic bacteria that determines the magnitude of pathological change in the established reservoir. In this study we could not show correlation between pathological scores or their distribution and any clinical parameter. In particular, there was no correlation between these scores and the duration of pouch functioning. These findings are supported by serial observation of reservoirs showing that the mucosa reaches a relatively steady state in terms of pathological changes soon after ileostomy reversal. ${ }^{17}$ Similar findings with all but one of the reservoirs ( $96 \%$ ) showing expression of at least one of these markers, there was relatively poor correlation between them. Fifty two per cent of reservoirs showed expression of one of the markers and not the other (Table III).

\section{Discussion}

Construction of a pelvic ileal reservoir with ileoanal anastomosis creates a functioning neorectum out of the terminal ileum. It is therefore not surprising that stasis and changes in faecal content lead to alterations in mucosal architecture and both acute and chronic inflammation. ${ }^{139}$ The enhanced changes observed in this study in posterior wall biopsy specimens compared with

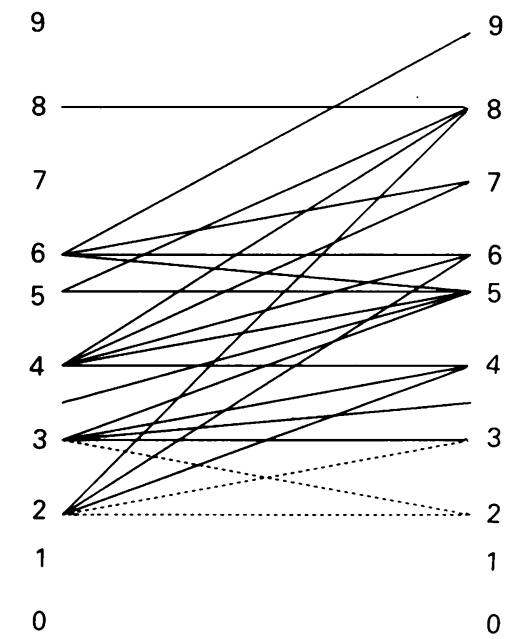

Anterior

Posterior

Figure 3: Comparison of anterior wall and posterior wall inflammatory scores in individual patients. The three hatched polyposis. lines represent the three patients with familial adenomatous 


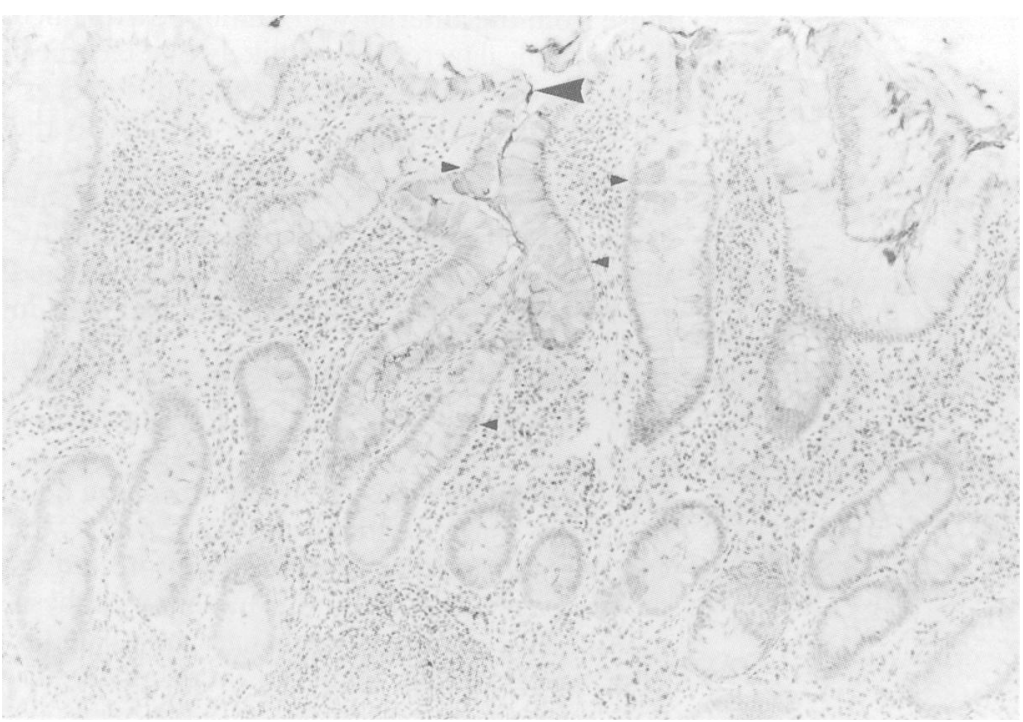

Figure 4: A pouch mucosal biopsy showing widespread expression of $P R 3 A 5$ in the goblet cells of the crypt epithelium (small arrowheads) and also in the extruded mucus (large arrowhead). Positive immunohistochemical staining manifests as grey staining within goblet cells and is particularly well seen in the central crypt below the large arrowhead. PR $3 A 5$ immunohistochemistry. (Haematoxylin and eosin, original magnification $\times 200$.) have been demonstrated in Kock's continent ileostomy reservoirs, ${ }^{18}{ }^{19}$ although there is some evidence to suggest reversion to almost normal intestinal mucosa occurs in these abdominal reservoirs after 15 years. ${ }^{20}$

Several divergent but characteristic pathological changes have been previously shown within the pouch mucosa. These include mucosal prolapse, pseudopyloric metaplasia, mucosal ischaemia, and granulomata. ${ }^{9}$ None of these features were seen in the current study despite extensive mucosal sampling, suggesting that all are relatively unusual. The pathological changes of mucosal prolapse deserve particular mention. A syndrome of anterior mucosal prolapse, so called anterior strip pouchitis, has been described with the characteristic pathological features of mucosal prolapse. ${ }^{9}$ We could not find any changes in any reservoir in anterior wall biopsy tissues, suggesting that this is a rare feature in the pouch. Inflammation in the proximal limb was also unusual. In the one patient in this series with this feature, there was marked active inflammation with expression of colonic phenotypic markers. Although clearly unusual, it would be important to document the presence of this inflammation as it may be of considerable significance to the surgeon considering pouch reconstruction on removal.

This study has shown that colonic phenotypic changes are very common in ileal reservoirs when several biopsy specimens are analysed. Sulphomucin expression has been seen in about $50 \%$ of reservoirs ${ }^{14}$ but these studies used only random biopsy tissues. The current studies show this mucin colonic phenotypic change in $61 \%$ of reservoirs and immunohistochemical colonic phenotypic change in $78 \%$, with only one reservoir failing to show expression of either of these markers. In $52 \%$ of pouches, however, there was expression of only one of these colonic phenotypic markers. In complete metaplasia there should be expression of all phenotypes native to that epithelium. ${ }^{21}$ Our findings suggest that either there is not true colonic metaplasia or that such a change is extremely focal. The restricted expression of colonic markers is probably the result of epigenetic alterations in the mucosal epithelium caused by changes in the environment. These findings are supported by the universal preservation in the pouch mucosa of small intestinal phenotypic expression as assessed by disaccharidase activity. ${ }^{+}$

A consistent feature of pelvic ileal reservoirs is the difference in inflammatory scores, particularly of active inflammation, between patients with ulcerative colitis and those with FAP.' In this study this difference is again apparent. This finding and the clinical and pathological evidence linking ulcerative colitis and pouchitis $^{91322}$ suggest an aetiopathogenetic link between the two conditions. Even so, pouchitis is an enigmatic condition and it may well be of multifactorial origin..$^{914}$ An interesting observation from the Gloucester series is the lack of any case fulfilling criteria for pouchitis despite a series of 55 well established reservoirs, including the 23 patients in the current study. These data suggest that technical factors may be responsible for some cases of pouchitis with mucosal ischaemia being a possible pathogenetic mechanism.

The reservoir mucosa shows enhanced proliferative activity both in patients with pouchitis and with those who do not have this relapsing chronic inflammatory condition of the reservoir. ${ }^{23}$ The coexistence of hyperproliferation and colonic phenotypic expression in the reservoir of patients with ulcerative colitis and FAP, conditions with high rates of neoplastic change in the large intestine, has led some to suggest that neoplastic change is a significant risk in the reservoir mucosa. ${ }^{92425}$ Despite the fact that very few reservoirs have been functioning for more than 10 years, cases have been described in which dysplasia ${ }^{25}$ and carcinoma ${ }^{26}$ have been seen in colitic pouches and adenomas in FAP pouches. ${ }^{127} 28$ It has been further postulated that pouchitis represents a recurrence of ulcerative colitis in reservoirs with colonic metaplasia. If, as our studies suggest, there is not true and complete colonic metaplasia, does this make these hypotheses any less likely? In the case of ulcerative colitis, the pathogenetic mechanisms may be directly related to one of the colonic phenotypic changes expressed in the ileal reservoir, such as mucin histochemical phenotype, and our evidence does not entirely refute the hypothesis that pouchitis is an expression of ulcerative colitis in the ileal reservoir. Given the predisposition of colonic mucosa for neoplasia in FAP, neoplastic change seems less likely than if the pouch environment approximated to that of the colon with complete metaplasia of the reservoir mucosa.

We are still uncertain of the risks of neoplastic change in the pouch and of the pathogenesis and long term importance of pouchitis. ${ }^{24}$ For this reason endoscopic and histopathological surveillance of the reservoir has been recommended. ${ }^{29} 30$ Our studies suggest that a single random biopsy of the mucosa is of limited value in the assessment of pathological changes and in identifying neoplastic change. Because of the demonstrable focality of pathological change, there is a need for standardisation of mucosal biopsy sites and this study clearly shows the importance of mucosal biopsy of the lower posterior wall with its 
susceptibility for more advanced inflammatory and morphological pathology.

1 Shepherd NA, Jass JR, Duval I, Moskowitz RL, Nicholls RJ, Morson BC. Restorative proctocolectomy with ilea reservoir: pathological and histochemical study of mucosal reservoir: pathological and histochemical study of

2 Moskowitz RL, Shepherd NA, Nicholls RJ. An assessment of inflammation in the reservoir after restorative proctocolectomy with ileoanal ileal reservoir. Int $\mathcal{F}$ Colorectal Dis 1986 1: $167-74$

3 O'Connell PR, Rankin DR, Weiland LH, Kelly KA. Enteric bacteriology, absorption, morphology and emptying after ileal pouch-anal anastomosis. Brf Surg 1986; 73: 909-14.

4 de Silva HJ, Millard PR, Kettlewell M, Mortensen NJ, Prince C, Jewell DP. Mucosal characteristics of pelvic ileal pouches. Gut 1991; 32: 61-5.

5 Corfield AP, Warren BF, Bartolo DCC. Mucins in ileo-anal pouches. BrF Surg (in press).

6 Shepherd NA. The pelvic ileal reservoir: pathology and pouchitis. Neth f Med 1990; 37: S57-64.

7 Bahia SS, MacMahon RFT, Hobbiss J, Taylor TV, Kelly KA, Carpenter HA. A histopathological assessment of ileo-anal reservoirs involving morphometric and histochemical characterisation $\mathcal{F}$ Pathol 1991; 164:363A.

8 Bruce D, Warren BF, Luckett M, Durdey P, Shepherd NA. The ultrastructural appearances of the pelvic ileal reservoir mucosa. Gut 1991; 32: A1218

9 Warren BF, Shepherd NA. Pouch Pathology. In: Nicholls RJ Mortensen NJMc, Bartolo DCC eds. Restorative proctocolectomy. Oxford; Blackwell Scientific Publications (in press)

10 Nicholls RJ, Pezim ME. Restorative proctocolectomy with ileal reservoir for ulcerative colitis and familial adenomatous polyposis: a comparison of three reservoir designs. BrF Surg polyposis: a compar

11 Spicer SS. The use of various cationic reagents in histochemical differentiation of mucopolysaccharides. Am $\mathcal{f}$ Clin chemical differentiation

12 Richman PI, Bodmer WF. Monoclonal antibodies to human colorectal epithelium. Markers for differentiation and tumour characterisation. Int $\mathcal{F}$ Cancer 1987; 39: 317-28.

13 Shepherd NA, Hulten L, Tytgat GNJ, et al Workshop: Pouchitis. Int $\mathcal{F}$ Colorectal Dis 1989; 4: 205-29.

14 Madden MV, Farthing MJG, Nicholls RJ. Inflammation in ileal reservoir: pouchitis. Gut 1990; 31: 247-9.

15 Nicholls RJ, Belliveau P, Neill M, Wilks M, Tabaqchali S Restorative proctocolectomy with ileal reservoir: a pathophysiological assessment. Gut 1981; 22: 462-8.
16 Nasmyth DG, Godwin PGR, Dixon MF, Williams NS, Johnston D. Ileal ecology after pouch-anal anastomosis or ileostomy. A study of mucosal morphology, fecal bacteriology, fecal volatile fatty acids and their interrelationship. Gastroenterologv 1989; 96: 81724 .

17 de Silva HJ, Millard PR, Prince C, Kettlewell M, Mortensen $\mathrm{N}$, Jewell DP. Serial observations of the mucosal changes in ileo-anal pouches. Gut 1990; 31: A1168-9.

18 Philipson B, Brandberg A, Jagenburg R, Kock NG, Lager I, Ahren C. Mucosal morphology, bacteriology and absorption in intra-abdominal ileostomy reservoir. Scand 7 Gastroenterol 1975; 10: 145-53.

19 Nilsson LO, Kock NG, Lindgren I, Myrvold HE, Philipson BM, Ahren C. Morphological and histochemical changes in the mucosa of the continent ileostomy reservoir 6-10 years after its construction. Scand 7 Gastroenterol 1980; 15: $737-47$.

20 Helander KG, Ahren C, Philipson BM, Samuelsson BM, Ojerskog BO. Structure of mucosa in continent ileal reservoirs 15 to 19 years after construction. Hum Pathol reservoirs 15 to 19

21 Slack JMW. Epithelial metaplasia and the second anatomy. Lancet 1986; ii: 268-70.

22 Tytgat GNJ, van Deventer SJH. Pouchitis. Int 7 Colorectal Dis 1988; 3: 226-8.

23 de Silva HJ, Gatter KC, Millard PR, Kettlewell M, Mortensen NJ, Jewell DP. Crypt cell proliferation and HLA-DR expression in pelvic ileal pouches. $\mathcal{F}$ Clin Pathol 1990; 43: $824-9$.

24 Shepherd NA. The pelvic ileal reservoir: apocalypse later? BMf 1990; 301: 886-7.

25 Lofberg R, Liljequist L, Lindquist K, Veress B, Reinholt FP, Tribukait B. Dysplasia and DNA aneuploidy in a pelvic pouch. Dis Colon Rectum 1991; 34: 280-4.

26 Stern H, Walfisch S, Mullen B, McLeod R Cohen Z. Cancer in an ileoanal reservoir: a new late complication? Gut 1990; 31: 473-5.

27 Beart RW, Fleming CR, Banks PM. Tubulovillous adenomas in a continent ileostomy after proctocolectomy for familial polyposis. Dig Dis Sci 1982; 27: 553-6.

28 Stryker SJ, Kent TH, Dozois RR. Multiple adenomatous polyps arising in a continent reservoir ileostomy. Int $\mathcal{F}$ Colorectal Dis 1987; 2: 43-5.

29 Tytgat GNJ. The role of endoscopy in pouch monitoring and pouchitis. In: Pouchitis Workshop. Int $\mathcal{f}$ Colorectal Dis $1989 ; 4: 210-3$.

30 Warren BF, Shepherd NA. The role of pathology in pouch surgery. Int $\mathcal{F}$ Colorectal Dis 1992; 7: 68-75. 